\title{
Self-Healing Polymer Composites Based on Hydrogen Bond Reinforced with Graphene Oxide
}

Yue Chen, ${ }^{1}$ Ying Wang, ${ }^{1}$ Tai Su, ${ }^{1}$ Jiayi Chen, ${ }^{1}$ Chao Zhang, ${ }^{1}$ Xiaoxing Lai, ${ }^{1}$ Dawei Jiang, ${ }^{1,{ }^{*}}$ Zijian Wu, ${ }^{3}$ Caiying Sun, ${ }^{1}$ Bin $\mathrm{Li}^{1,2^{*}}$ and Zhanhu Guo ${ }^{4 *}$

A self-healing supramolecular polymer composite (LP-GO) is designed and prepared via incorporation of graphene oxide (GO) to hyperbranched polymer by hydrogen-bonding interactions. The polymer matrix based on amino-terminated hyperbranched polymer is synthesized by dimer acid and diethylene triamine, while GO is prepared by the modified Hummers method. Infrared spectroscopy (FTIR), thermo gravimetric testing (TGA), X-ray diffraction (XRD), and scanning electron microscopy (SEM) is applied to characterize GO. Stressstrain test is utilized to characterize the obtained self-healing property of LP-GO. It is found that just a small amount of GO (up to 3 wt \%) is needed to achieve a dramatic improvement in the mechanical properties, and self-healing efficiency of the polymer composites. After healing at $60{ }^{\circ} \mathrm{C}$ for $1 \mathrm{~h}$, the addition of $\mathrm{GO}$ even restores the self-healing efficiency to $100 \%$ of its original tensile strength. In striking contrast to conventional cross-linked or thermoreversible rubbers made of macromolecules, these systems, when being broken or cut, can be simply healed by contacting fractured areas again to self-heal at suitable temperature. Building on the unique self-healing properties, the simplicity of synthesis and the availability from renewable resources, etc., LP-GO bodes well for broader applications in the near future.

Keywords: Self-healing; Polymer composite; Graphene oxide; Hydrogen bond

Received 1 February 2019, Accepted 15 March 2019

DOI: $10.30919 /$ esmm5f214

\section{Introduction}

The organic polymers have found a wide utilization in diverse fields, such as textiles, packaging, automobiles, etc., owing to the characteristics of low cost and easy processing making them to be the basic materials in modern life. ${ }^{1-3}$ In recent years, as significant efforts have been emphasized on the exploitation of novel polymers with enhanced functionality, including biodegradability, ${ }^{4,5}$ semiconductivity, electrical conductivity and self-healing capacity, ${ }^{6-8}$ the usage of organic polymers has been developed rapidly, in which cross-linked polymers, such as epoxy resin and phenolic resin, have strong mechanical properties but relatively fragile leading to irreparable fracture or breakage under a certain amount of external force during processing and employing, so that applications of this kind of polymers are often subjected to limitation. ${ }^{9,10}$ In view of that self-healing can effectively extend the life of materials, realize recycling and reusing, and reduce a string of security issues and environmental problems invited by material

${ }^{1}$ College of Science, Northeast Forestry University, Harbin 150040, China

${ }^{2}$ Post-doctoral Mobile Research Station of Forestry Engineering, Northeast Forestry University, Harbin 150040, China

${ }^{3}$ Key Laboratory of Engineering Dielectrics and Its Application, Ministry of Education, Harbin University of Science and Technology, Harbin 150040, China

${ }^{4}$ Integrated Composites Laboratory (ICL), Department of Chemical Engineering, University of Tennessee, Knoxville TN 37996, USA

*E-mail:daweijiang@nefu.edu.cn; libinzh62@163.com;

zguo10@utk.edu failure, the research on self-healing polymers is of great significance. ${ }^{11-15}$ The self-healing polymers can be divided into two categories, according to the different preparation methods. One is extraneous self-healing polymers, that is, the materials can realize the restoration of mechanical properties and integrity without external heating and other stimulation through embedding healing reagents into substrate. ${ }^{16,17}$ White et al ${ }^{18}$ first proposed microcapsule self-healing technology which has been widely utilized and developed. The cyclopentine diocene is wrapped in a microcapsule made from the urea formaldehyde resin before it is dispersed in diethylenetriamine cured epoxy resin oligomer till in touch with the Grubbs catalyst. When cracks are generated in the material, the microcapsules are broken, and the cyclopentadiene dimer, in the meantime, rapidly infiltrate into the cracks due to capillary siphonage. When it comes into contact with Grubbs catalyst, the active ringopening polymerization reaction occurs, and a highly cross-linked polymer network is created in large quantity to achieve the purpose of healing. Only, this kind of healing technology needs additional healing agents, realize anything but single self-healing. Moreover the release of healing agents forms new voids which will turn into new defects of the materials. The other is intrinsic self-healing polymers based on reversible bonds including dynamic covalent or non-covalent bonds. Unlike the extraneous self-healing polymers, numerous dynamic chemical bonds or weak interactions are required to self-heal instead of additional healing reagents. ${ }^{19,20}$ Theoretically, it is the main characteristic that intrinsic self-healing polymers have the ability to bring about multiple self-heal of materials, although its application is frequently restricted by the demand of external conditions such as heating, ${ }^{21,22}$ lighting, ${ }^{23,24}$ and electromagnetic effects ${ }^{25}$ to self-healing. At present, the supramolecular polymers based on hydrogen bonding networks provide a promising access towards spontaneous self-healing polymers for the 
swift self-healing accomplished at low temperature. ${ }^{26}$ Hence, Leibler's ${ }^{27}$ group reported a self-healing polymer composed of multifunctional fatty acids and urea compounds, as well as associated by molecular hydrogen bonding. If the material suffers damage, the hydrogen bonds tend to break first owing to the weak strength so that the non-associated groups will appear in the fractured area, unless new hydrogen bond association can be produced again at room temperature after bringing the two broken pieces gently back into contact. However, the addition of an $11 \% \mathrm{w} / \mathrm{w}$ plasticizer, necessary to lower the high glass transition temperature of obtained self-healing polymers, restrains the movement of the polymer chains bearing hydrogen bonds, which always results in a slow mechanical healing speed (typically over one day for complete healing). To address this problem, Wang et $a l^{28}$ synthesize a self-healing nanocomposite by means of using modified graphene oxide (GO) that is treated with thionyl chloride as macroscopic crosslinking agents. When the weight percentage of modified GO changes from $1 \%$ to $4 \%$, the tensile strength changes from $0.26 \mathrm{MPa}$ to $0.8 \mathrm{MPa}$. As the above data, the relatively poor mechanical properties make nanocomposites unsuitable for practical applications in high strength domains. Similarly, Luan et $a l^{29}$ introduce GO modified by adding 2, 4-tolylene diisocyanate and hydroxyl terminated hyperbranched polyester into supramolecular polymer based on amino-terminated hyperbranched polymer which is synthesized from the hydroxyl-terminated hyperbranched polyester, succinic anhydride, diphenyl azidophosphate, and diethylenetriamine as the raw materials, and then obtained the self-healing elastomer via filling $1 \mathrm{wt} \%$ or $2 \mathrm{wt} \%$ modified GO, respectively. Nevertheless, when the content of modified GO is $2 \mathrm{wt} \%$, the maximum mechanical strength is less than 0.6 MPa. In these two examples, though the healing speed of the supramolecular polymers increases and the glass transition temperature decreases, the small fraction between grafted groups on the surface of GO lowers the cross-linking degree of modified GO and polymers, and reduces the mechanical properties, thus constrains the high-strength applications as in sports equipments, ballistic armors, and the aerospace industry. Hence, developing materials that possess parameters including adequate mechanical strength and quick selfhealing speed, still faces daunting challenge.

GO has excellent properties, such as high mechanical strength, high surface area and abundant functional groups, ${ }^{30-32}$ attractive to add it into the self-healing materials. By this way, GO can better integrate with the organic substrate and produce functional materials with excellent mechanical properties. ${ }^{33,34}$ In this paper, the modified Hummers method ${ }^{35}$ is implemented to prepare GO, not only has more hydroxyl groups and carboxyl groups but contains a certain amount of epoxy groups. The incorporation of low content of reinforcement into the polymer system exerts a negligible effect on the density of hydrogen bonds or the movement of molecular chain containing the hydrogen bonds in that a spot of GO, even though, could significantly improve the mechanical property of self-healing polymers.

To investigate the influence of $\mathrm{GO}$, as a reinforcement, on the mechanical properties in amino-terminated hyperbranched polymers, pure self-healing polymers (LP) and self-healing polymers containing different weight percentage of $3 \mathrm{wt} \%, 5 \mathrm{wt} \%, 7 \mathrm{wt} \%$ and $9 \mathrm{wt} \%$ of GO, termed as LP, LP-3, LP-5, LP-7 and LP-9, are synthesized. Through the mechanical property test, it is discovered that the mechanical property of the self-healing polymers gradually decreases with the increasing proportion of GO, and the mechanical strength is much higher than that of LP overall. The optimum addition ratio of the reinforcement is $3 \mathrm{wt} \%$ and the tensile strength is up to $2.95 \mathrm{MPa}$. From the healing cycle test under various healing conditions, optimal healing condition of LP-3 can be obtained by comparing the healing efficiency. After two broken pieces contact for $3 \mathrm{~h}$ at $30{ }^{\circ} \mathrm{C}$, the LP-3 sample heals to $50 \%$ of its original tensile strength, while the span of time will be shortened to $2 \mathrm{~h}$ when the temperature is $40{ }^{\circ} \mathrm{C}$. Mechanical healing can be completed in $6 \mathrm{~h}$ at $50{ }^{\circ} \mathrm{C}$ while $1 \mathrm{~h}$ is enough at $60{ }^{\circ} \mathrm{C}$, which means shorter healing time matches with higher healing temperature or lower healing temperature acquires longer healing time. Even if 10 fracture-healing cycles under the appropriate healing condition, the mechanical strength of LP-3 can still be restored to $100 \%$, which verifies the self-healing polymer composites (LP-GO) have a lower descent rate of healing efficiency compared with LP, that can be attributed to the loss of hydrogen bonds sites in the composites. The thermodynamic stability of LP-3, on account of the TGA\&DTG analysis, is also greatly improved.

\section{Experimental Section}

\subsection{Materials}

Empol 1016 high pure Dimer Acid was purchased from BASF China ( $80 \%$ dibasic acids, $16 \%$ polybasic acids, $4 \%$ monobasic acids). The AR diethylenetriamine was available from Sigma Aldrich. Flake graphite (FG) was obtained from Qingdao Graphite Products Co., China. Chloroform, methyl alcohol, ethanol, and urea were supplied by Tianjin Tianli Fine chemical Co., Ltd. , China. All the chemicals can be applied directly without further processing.

\subsection{Preparation of materials}

\subsubsection{Preparation of $\mathrm{GO}$}

The synthesis principle of GO, abiding by the modified Hummer's methods, is described in detail in the following reaction equations. The preparation process is divided into three stages: low temperature intercalation, medium temperature oxidation and high temperature hydrolysis stripping. In the intercalation stage, since concentrated sulfuric acid as intercalation agent cannot spontaneously react with FG, the chemical oxidation method should be applied - as the first react equation shows that $\mathrm{KMnO}_{4}$ reacted with cold concentrated sulfuric acid at low temperature to generate the oxidative active component $\mathrm{Mn}_{2} \mathrm{O}_{7}$ to slightly oxidize the edge and defects of FG and increase the interlayer spacing for intercalation of sulfuric acid and sulfate ion. In the oxidation stage, with the ascension of temperature, the strong oxidation effect of the oxidant gave rise to a large amount of oxidation of FG, which not only formed oxygen-containing functional groups on the surface, but continued increasing the interlayer spacing, as described in the second reaction equation. In the stripping phase, as the appropriate content of hydrogen peroxide was added to the reactants, the unreacted oxidant was reduced to colorless soluble $\mathrm{MnSO}_{4}$, demonstrated in the third equation, and then GO was stripped by pickling, washing and ultrasound. Specifically, $1.0 \mathrm{~g}$ of FG, $10 \mathrm{~mL}$ and $90 \mathrm{~mL}$ of sulfuric acid and phosphate (1:9 by volume) were mixed with $6 \mathrm{~g}$ of potassium permanganate, and stirred in the $4{ }^{\circ} \mathrm{C}$ ice water bath for $1 \mathrm{~h}$ and at $50{ }^{\circ} \mathrm{C}$ for $24 \mathrm{~h}$ until a homogeneous dispersion was attained. The dispersion was put into ice water and blended dropwise with volume fraction $30 \%$ of hydrogen peroxide to turn it golden. The resulted mixture was washed successively with volume fraction $5 \%$ of dilute hydrochloric acid solution and distilled water until the supernatant was neutral by centrifugation. The clean and sticky sendiment was sonicated for $1 \mathrm{~h}$ before the coarse product dried at $70{ }^{\circ} \mathrm{C}$ under vacuum (Fig. 1).

$$
\begin{aligned}
& \mathrm{KMnO}_{4}+\mathrm{H}_{2} \mathrm{SO}_{4} \rightarrow \mathrm{K}_{2} \mathrm{SO}_{4}+\mathrm{Mn}_{2} \mathrm{O}_{7}+\mathrm{H}_{2} \mathrm{O} \\
& \mathrm{KMnO}_{4}+\mathrm{H}_{2} \mathrm{SO}_{4}+\mathrm{C} \rightarrow \mathrm{MnSO}_{4}+\mathrm{CO}_{2}+\mathrm{SO}_{2}+\mathrm{H}_{2} \mathrm{O} \\
& \mathrm{KMnO}_{4}+\mathrm{H}_{2} \mathrm{SO}_{4}+\mathrm{H}_{2} \mathrm{O}_{2} \rightarrow \mathrm{MnSO}_{4}+\mathrm{K}_{2} \mathrm{SO}_{4}+\mathrm{H}_{2} \mathrm{O}+\mathrm{O}_{4}
\end{aligned}
$$




\subsubsection{Preparation of oligomers}

A mixture of dimer acid $(30 \mathrm{~g})$ and $12.3 \mathrm{~g}$ of diethylene triamine were stirred at $160{ }^{\circ} \mathrm{C}$ for $24 \mathrm{~h}$ under nitrogen atmosphere. Then the mixture was extracted three times by chloroform $(50 \mathrm{~mL})$ in the separation funnel. When the dispersion statically separated and the equilibrium was reached, the lower layer was released and the upper layer was washed with $90 \mathrm{~mL}$ of the mixture methanol and water (1:2 by volume) to obtain the dendritic oligomer (Fig. 1).

\subsubsection{Preparation of self-healing polymers}

$1.18 \mathrm{~g}$ of urea was slowly added to $5 \mathrm{~g}$ of oligomer and kept stirring up to $100{ }^{\circ} \mathrm{C}$. When heating to $135{ }^{\circ} \mathrm{C}$, increase temperature at the rate of 5 ${ }^{\circ} \mathrm{C}$ each hour till $160{ }^{\circ} \mathrm{C}$. The obtained LP was then molded onto the samples for testing. For LP-3, extra $0.15 \mathrm{~g}$ of GO was considered to mix with $5 \mathrm{~g}$ oligomer apart from other reaction steps. Similarly, LP-5, LP-7 and LP-9 were obtained from mixing $5 \mathrm{~g}$ oligomer with $0.25 \mathrm{~g}$, $0.35 \mathrm{~g}$ and $0.45 \mathrm{~g}$ of GO, respectively, in the same way. (Fig. 1).

\subsection{Characterization}

Infrared characterization of the molecular structure of natural flake graphite (FG) and GO samples were performed by means of the Fourier transform infrared spectrometer (Perkin Elmer, Spectrum 400, USA) with a scan range of 400-4000 $\mathrm{cm}^{-1}$. The contents of functional groups were conducted by XRD (Shimadzu, XRD-6100, Japan). Thermal stability was acquired from TGA\&DTG (Perkin Elmer, Pyris1, USA). The mechanical tensile stress test was carried out with a WDW-2 type microcomputer controlled electronic universal testing machine (Jinan Yongke Testing Instrument Co., Ltd.). The thermal stability of LP and LP-GO was compared via using a thermogravimetric analyzer (Perkin Elmer, Pyris 1, USA) as well. Their surface topography was performed by scanning electron microscopy (SEM) (Hitachi, Su8020).

\section{Results and discussion}

\subsection{Molecular structure and properties of GO \\ 3.1.1 Surface groups of $G O$}

Fig. 2a shows the infrared spectra of FG and GO. As can be seen from the curve of FG, the characteristic absorption peaks of the functional groups of FG are relatively weak, and the infrared spectrum curve is rather smooth, compared to the curve of GO in which the chemical structure can be definitely analyzed. In the vicinity of the high frequency region $3433 \mathrm{~cm}^{-1}$, the stretching vibration assigned to $-\mathrm{OH}$ may comes from water molecules adsorbed by GO because of the strong hygroscopicity. The absorption peaks near the $2928 \mathrm{~cm}^{-1}$ and $2861 \mathrm{~cm}^{-1}$ positions correspond to the antisymmetric and symmetric stretching vibrations of $\mathrm{CH}_{2}$, respectively, and near the position of 1625 $\mathrm{cm}^{-1}$ in the intermediate frequency region belongs to the $\mathrm{C}=\mathrm{O}$ stretching vibration of carboxylic acid and carbonyl on the edge of GO. The C-O stretching vibration of the carboxyl group emerges at $1394 \mathrm{~cm}^{-1}$ and the C-O-C stretching vibration of GO surface is attributed near the position of $1117 \mathrm{~cm}^{-1}$. In summary, the presence of these oxygen-containing groups indicates that FG has been oxidized and these polar groups, especially the hydroxyl groups on the surface, make GO liable to form hydrogen bonds with water molecules, thereby explaining the strong hydrophilicity of GO

\subsubsection{Thermal properties of $G O$}

As shown in the Fig. 2b, the thermal stability of FG is significantly higher than that of GO. FG does not exhibit a substantial loss of mass until above $600{ }^{\circ} \mathrm{C}$, and before that, it shows good thermal stability, by contrast, the weight loss process of GO can be divided into three stages within the scope of the test temperature: $50 \sim 165{ }^{\circ} \mathrm{C}, 165 \sim 230{ }^{\circ} \mathrm{C}$ and above $230{ }^{\circ} \mathrm{C}$. A thermogravimetric platform between 50 and $165{ }^{\circ} \mathrm{C}$ is mainly due to the gasification of a little water adsorbed on the surface of GO. After $165^{\circ} \mathrm{C}$ is the major part of the weight loss contributed to the gasification of the bound water between the GO layers and the conversion of some reactive groups into carbon dioxide during the
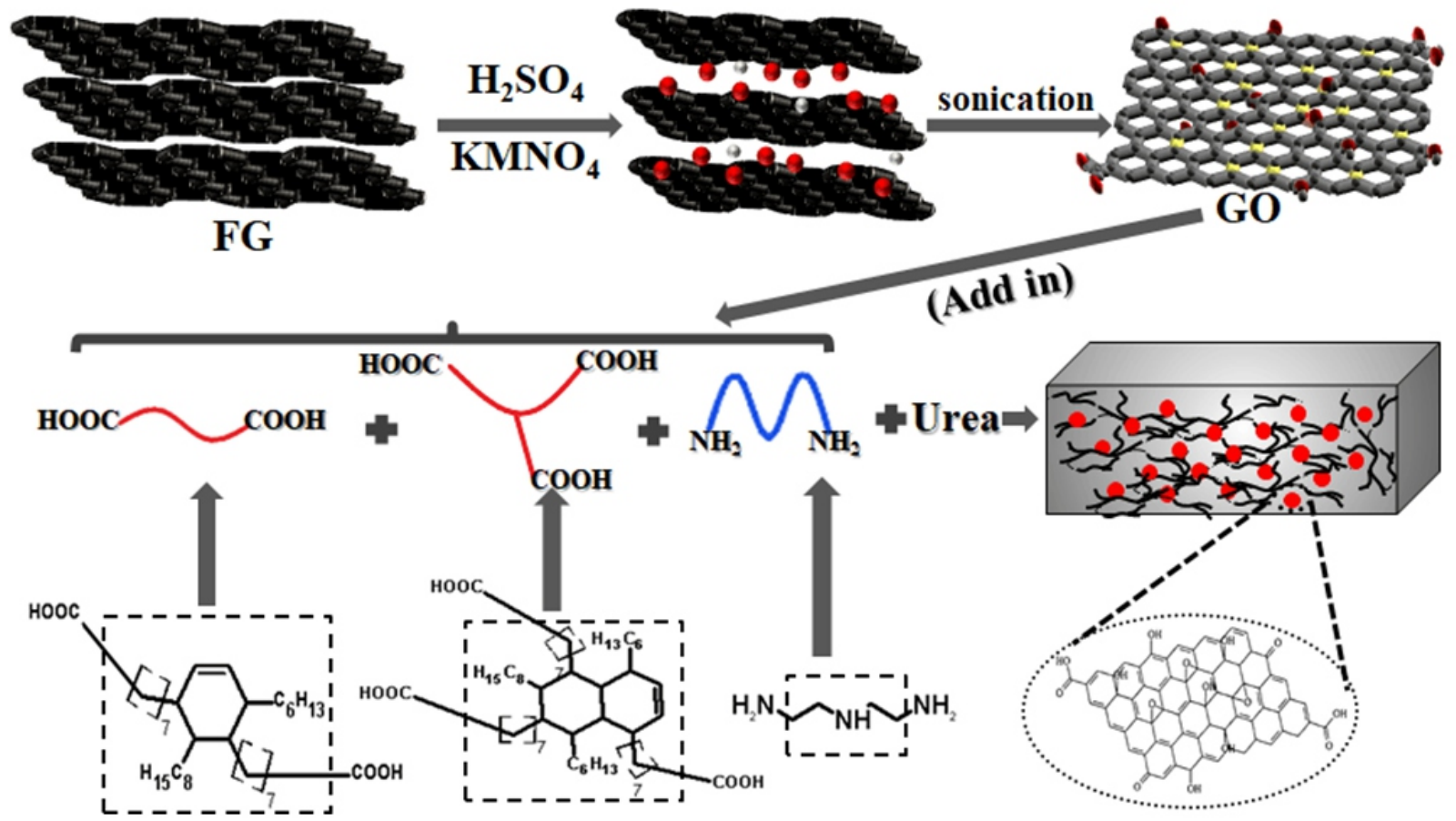

(Add in)

Fig. 1 Schematic diagram of reaction mechanism and synthesis of LP-GO. 
heating process. Above $230^{\circ} \mathrm{C}$, the weight loss of the sample gradually drops to constant which boils down to the dissociation of the remaining oxygen molecules.

\subsubsection{Molecular structure of $G O$}

The XRD patterns of FG and GO can be found in Fig. 2c. Among them, FG has a sharp, high-intensity diffraction peak at $2 \theta$ of $26.6^{\circ}$, which is the characteristic diffraction peak of FG and also demonstrates the spatial structure of FG sheets is extremely arranged neatly.

The other shows the XRD pattern of GO of which diffraction peak turns very small except a relatively strong diffraction peak at $2 \theta$ of $11.3^{\circ}$ that indicates the structure of FG has been destroyed, the interlayer spacing is larger than that of FG and a new crystal structure has been in shape.

\subsubsection{Microscopic topography and element content of GO}

Fig. 3a exhibits the high resolution SEM image of the stacked FG that agglomerates in a few places, and the multilayered structure can be observed clearly. In comparison, GO possesses a thin sheet structure, which has apparent wrinkles and edge curling as confirmed by the SEM result (Fig 3b). The horizontal size of the GO nanosheets is between several hundred nanometers and several micrometers. While Fig $3 \mathrm{~b}$ can not accurately characterize the thickness of the GO nanosheets, the thickness of the FG can be estimated from a few nanometers to several tens of nanometers from the width of the edge of the sheet and the width of the wrinkles. Hence, the samples prepared via the modified Hummers method have topographical characteristics of GO. The bonding status of the $\mathrm{C}$ element existing in $\mathrm{GO}$ are confirmed by XPS (Fig. 3c). According to the fitting analysis of the $\mathrm{C}$ element peak, the $\mathrm{C}$ element mainly consists of $\mathrm{C}-\mathrm{O}$ bond (content $48.32 \%$ ), C-C bond (content 24.95\%), $\mathrm{C}=\mathrm{O}$ bond (content $19.18 \%$ ) and $\mathrm{O}-\mathrm{C}=\mathrm{O}$ bond (content $7.55 \%$ ). It is demonstrated that the polyaromatic ring system is modified by various oxygen-containing functional groups after the process of FG.

\subsection{Property analysis of self-healing polymers \\ 3.2.1 Thermal properties of $L P-G O$}

In order to maintain a reversible self-healing system, it is necessary to ensure that each component in the system is thermally stable during self-heal, namely no volatilization or degradation occurs. To measure and compare the relationship between LP and LP-GO when temperature changes in a nitrogen atmosphere, TGA\&DTG analysis is taken into account. As the Fig. 4a, mass loss of LP and LP-GO among $0 \sim 197^{\circ} \mathrm{C}$ are all about 3.6\%, nevertheless error in the allowable scope can be thought as no quality loss between $0 \sim 197{ }^{\circ} \mathrm{C}$ due to the instrument operation error and repeated use of the crucible, note that, which provides necessary conditions for self-heal to repeat many times. In addition, the rate of weight loss of LP-3 is slower relative to LP, indicating that the thermal stability of LP-3 is higher.

\subsubsection{Mechanical property of $L P-G O$}

To verify the good compatibility of GO with self-healing polymers and obtain suitable addition ratio of GO, the tensile test on self-healing polymers is carried out at $20^{\circ} \mathrm{C}$ in a strain rate of $1 \mathrm{~mm} / \mathrm{min}$. First, the
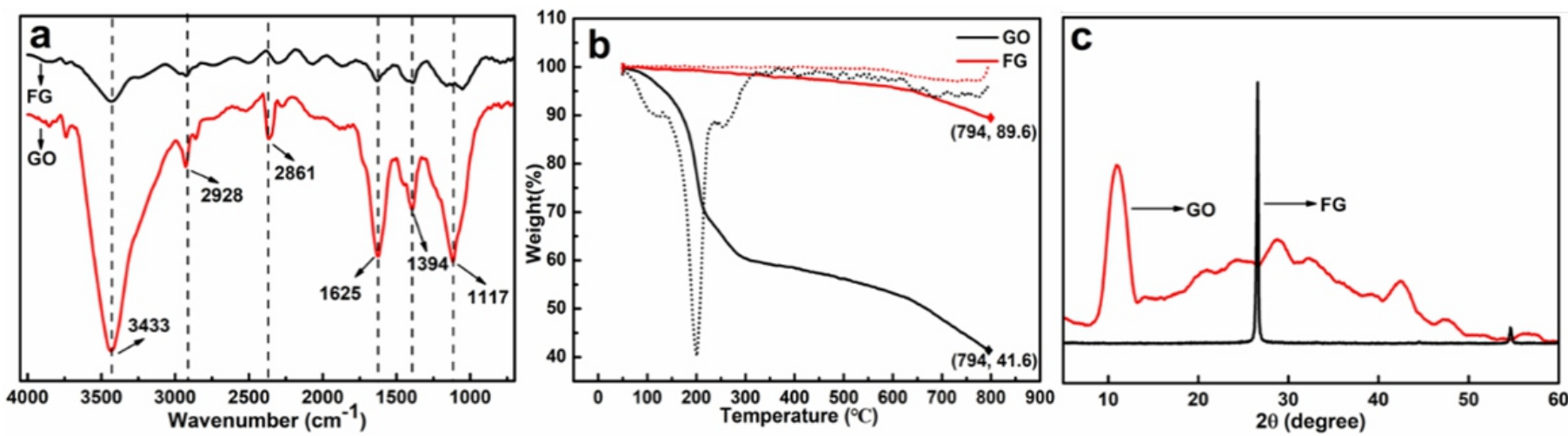

Fig. 2 a FTIR, b TGA\&DTG, $\mathbf{c}$ XRD curves of GO and FG.
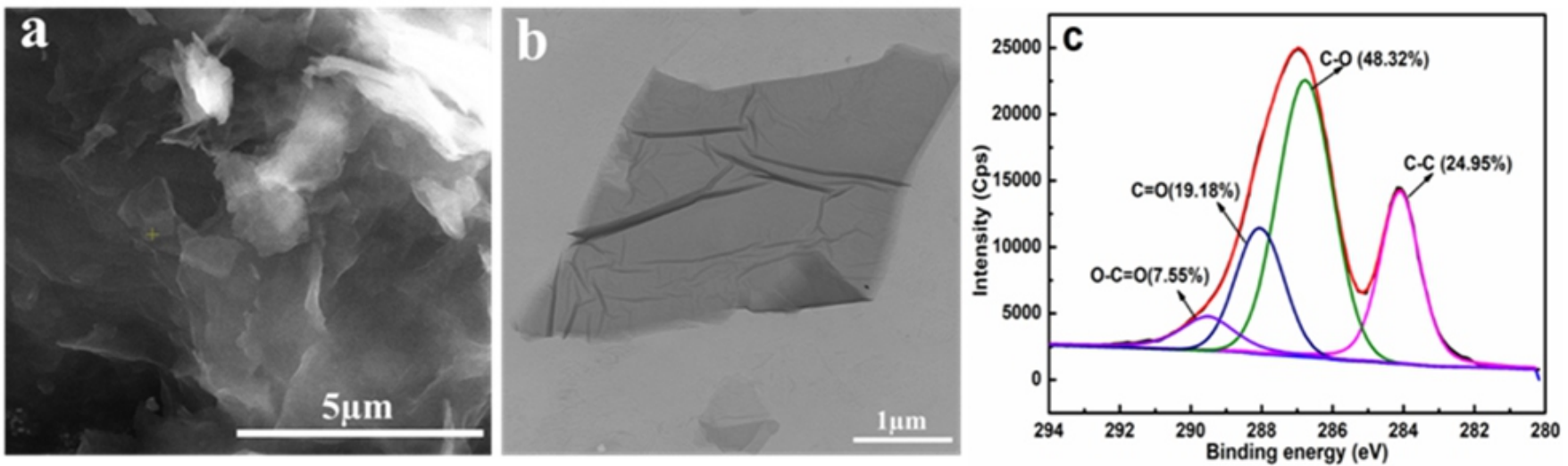

Fig. 3 a, b SEM images of FG and GO, c XPS spectra of GO. 
self-healing material is compacted with a pneumatic tablet press machine, and then cut into several groups of regular and flat splines with a blade. At the very least 6 samples are measured for each mass fraction of LP-GO. The tensile tester uniformly stretches the sample to obtain a smooth tensile profile and a series of data of tensile strength involving at break. Through performing a large number of parallel tests on each set of samples, the average tensile strength of each group is calculated, and the relationship between the addition ratio and the tensile strength is obtained. Detailed mechanical properties of LP and LP-GO composites are manifested in Fig. 4b. From the stress-strain curves of all materials, it is evident that LP-9 has largest strain at break up to $14 \%$ while LP is nearly $0.5 \%$. To put it another way, the former is more elastic while the latter is brittle which can be transformed by applying different content of GO. The variation trend of mechanical properties are shown in Fig. 4c, that the tensile strength of LP-GO increases first and then decreases along with the increase of the addition ratio of GO. In specific, when the GO addition ratio is $3 \mathrm{wt} \%$, LP-GO has maximum tensile strength at break up to $2.95 \mathrm{MPa}$, namely the optimum addition of GO is $3 \mathrm{wt} \%$. It can be seen that the breaking stress (2.95 MPa) of LP-3 increases over four times than LP $(0.7 \mathrm{MPa})$. When the weight percentage of GO changes from $3 \mathrm{wt} \%$ to $9 \mathrm{wt} \%$, the maximum strain decreases from 2.95 $\mathrm{MPa}$ to $1.02 \mathrm{MPa}$. On the one hand, the possible reason for the enhancement in mechanical strength of LP-GO before the GO addition ratio is less than $3 \mathrm{wt} \%$, is that the quantity of oxygencontaining functional groups gradually increases as the proportion of addition ascends, making GO more compatible with self-healing polymers. Besides, lower content allows GO to evenly disperse in the composite material, which makes the stress distribution of the system uniform, thus the tensile properties and the elasticity of the LP-GO are enhanced. On the other hand, when the addition ratio exceeds $3 \mathrm{wt} \%$, the break strain of LP-GO descends, which can be ascribed to the fact that GO nanosheets disturb the orientations of the polymer chains at high elongations, and consequently reduce the mobility of molecular chains.

\subsubsection{Self-healing property of $L P-G O$}

The healing properties are further tested by measuring the self-healing efficiency of LP-3 with the best mechanical properties. The two broken pieces are first closely contacted with each other and heal at $30^{\circ} \mathrm{C}$ for 1 $\mathrm{h}$ under vacuum. Subsequently, tensile strength after healing is measured and the healing efficiency is calculated by the ratio between tensile strength of the healing sample and the original sample. The span of healing time is prolonged to $2 \mathrm{~h}, 3 \mathrm{~h}$ and $6 \mathrm{~h}$ so as to explore the relationship between the healing time and healing efficiency, as demonstrated in Fig.5a, that the healing efficiency gradually increases as the growth of healing time under the constant temperature, and higher healing temperature comes with higher healing efficiency within the same healing time among $30{ }^{\circ} \mathrm{C}, 40{ }^{\circ} \mathrm{C}, 50{ }^{\circ} \mathrm{C}$ and $60{ }^{\circ} \mathrm{C}$. Moreover, a conclusion is clearly shown in Fig. $5 \mathrm{a}$ that the healing samples can reach the original mechanical properties at $60{ }^{\circ} \mathrm{C}, 1 \mathrm{~h}$ and $50{ }^{\circ} \mathrm{C}, 6 \mathrm{~h}$, confirmed by the data listed in the Table 1 or the tensile strain-stress curves in Fig. 5c below on the mechanical properties of the original LP3 and self-healing LP-3. In view of shortening the process time, the condition of $60^{\circ} \mathrm{C}$ for $1 \mathrm{~h}$ is adopted to perform the healing cycle test.

Furthermore, undergoing 10 fracture-healing cycle tests at $60^{\circ} \mathrm{C}$ for $1 \mathrm{~h}$, though LP-3 still completely restores the original mechanical properties each fracture-healing cycle later shown in Fig. 5b, which proves the reversibility and stability of LP-GO healing performance.

From Fig. 6d to Fig. 6f, illustrate the process, in part, of the fracture-healing cycle, and characterize the healing samples via SEM, of which images affirms that the physical interface at the fracture has been sufficiently healed such that the gap is indiscernible (Fig. 6b) in contrast to the pristine surface in the identical position (Fig. 6a). Obviously, the resulting healed sample can be subsequently subjected to $100 \%$ strain, which proves that GO as reinforcement has small effect on the selfhealing performance of LP-GO.
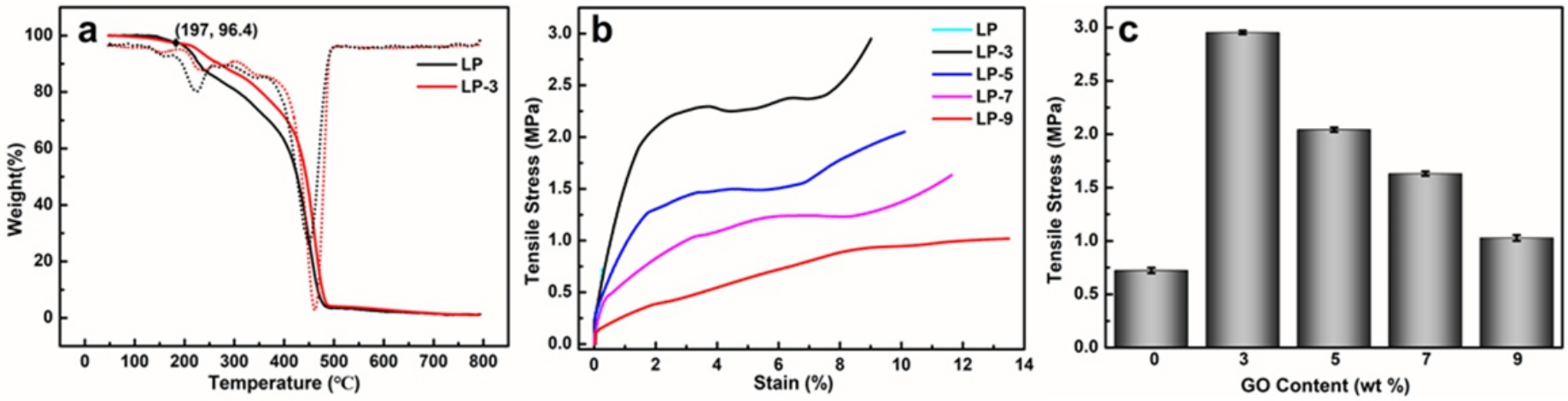

Fig. 4 a TGA\&DTG curves of LP and LP-3, b, $\mathbf{c}$ The tensile strain-stress curves and tensile stress of LP-GO with different GO contents.

Table 1 Summary of the mechanical properties of the original LP-3 and self-healing LP-3.

\begin{tabular}{cccc}
\hline Sample & $\begin{array}{c}\text { Young's Modulus } \\
\text { (MPa) }\end{array}$ & $\begin{array}{c}\text { Strain -at-break } \\
(\mathbf{\%})\end{array}$ & $\begin{array}{c}\text { Stress -at-break } \\
\text { (MPa) }\end{array}$ \\
\hline Original LP-3 & 32.74 & 9.01 & 2.9503 \\
Self-healing LP -3 & 33.48 & 8.81 & 2.9497 \\
\hline
\end{tabular}




\section{Conclusions}

In conclusion, the self-healing polymer composite (LP-GO) is prepared by supramolecular polymer matrix with hydrogen bonds and appropriate proportion of GO, which not only has good self-healing properties (more hydrogen bond binding sites, as can be verified from the FTIR, TGA\&DTG, XRD and SEM test analysis of GO) and thermal stability (TGA test of self-healing polymers), but effectively improves the defects of poor mechanical properties in traditional self-healing polymers. In addition, the optimal addition ratio of GO is determined 3 $\mathrm{wt} \%$ through the mechanical tensile stress test (more than this ratio is prone to agglomerate, less than is difficult to polymerize). The appropriate healing condition is at $60{ }^{\circ} \mathrm{C}$ for $1 \mathrm{~h}$, and the excellent healing effect can be visually reflected from the SEM image. Eventually, the fracture-healing cycles is carried out under the appropriate healing condition to verify that the stability of the healing performance of LP-GO conforms to the conclusion that the intrinsic self-healing polymers can theoretically realize the infinite healing cycles.

There is no doubt that the preparation of LP-GO still has large space for further development, for instance, the preparation of highquality single-layer GO and the compatibility of GO with polymers, etc., whereas in contrast to the self-healing system reported previously,
LP-GO system does not require healing agents, plasticizers or solvents and enhances the mechanical property. Most importantly, the material may have a promising application prospects depending on these properties.

\section{Conflicts of interest}

There are no conflicts of interest to declare.

\section{Acknowledgements}

Supported by Heilongjiang Natural Science Foundation (QC2017038), Fundamental Research Funds for the Central Universities (No. 2572018BC27), China Post-doctoral Science Foundation (No. 2016M601402 \&2017M610212), Heilongjiang Postdoctoral Fund (No. LBH-Z16004, LBH-Z16089), and Heilongjiang Postdoctoral Special Fund (No. LBH-TZ1713), University Research Training Program of Northeast Forestry University (KY2018016).

\section{Reference}

1. C. G. Jayalakshmi, A. Inamdar, A. Anand and B. Kandasubramanian, J. Appl. Polym. Sci., 2019, 136, 47241.

2. M. Naseri, L. Fotouhi and A. Ehsani, Chem. Rec., 2018, 18, 599-618.
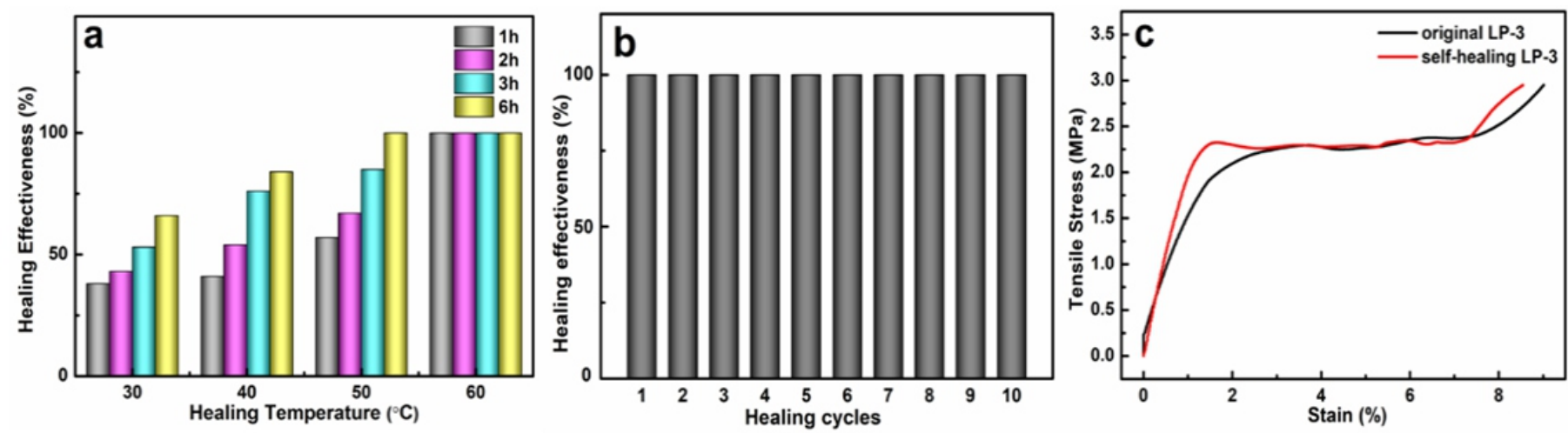

Fig. 5 a Self-healing efficiency under different conditions of LP-3, b The self-healing efficiency of LP-3 versus healing cycles, $\mathbf{c}$ The tensile strainstress curves of the original LP-3 and self-healing LP-3.
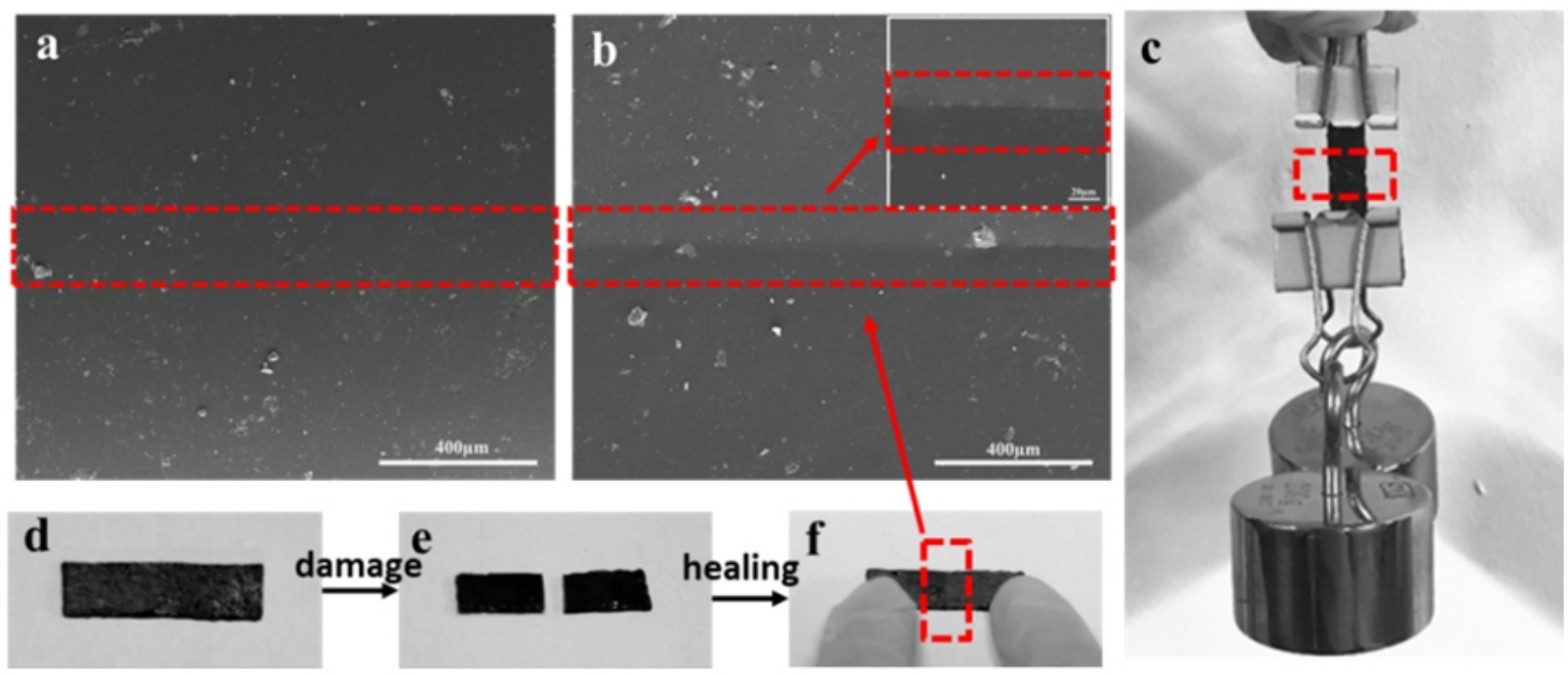

Fig. 6 a, b SEM images of surface of original and self-healing LP-3, Inset b: SEM images of larger magnification, $\mathbf{c}$ the healing sample under $300 \mathrm{~g}$ of weight $\mathbf{d}$, e, f Photographs portraying the self-healing of the nanocomposite with LP-3. 
3. Z. Ma, S. Li, H. T. Wang, W. Cheng, Y. Li, L. J. Pan and Y. Shi, J. Mat. Chem. B., 2019, 7, 173-197.

4. Q. Q. Miao, C. Xie, X. Zhen, Y. Lyu, H. W. Duan, X. G. Liu, J. V. Jokerst and K. Y. Pu, Nat. Biotechnol., 2017, 35, 1102-1110.

5. J. D. Shao, H. H. Xie, H. Huang, Z. B. Li, Z. B. Sun, Y. H. Xu, Q. L. Xiao, X. F. Yu, Y. T. Zhao, H. Zhang, H. Y. Wang and P. K. Chu, Nat. Commun., 2016, 7, 12967.

6. S. S. Wang, L. N. Li, Z. H. Sun, C. M. Ji, S. J. Liu, Z. Y. Wu, S. E. Zhao, A. Zeb, M. C. Hong and J. H. Luo, J. Mater. Chem. C., 2017, 5, 4731-4735.

7. J. R. Mou, Y. L. Deng, L. H. He, Q. J. Zheng, N. Jiang and D. M. Lin, Electrochim. Acta, 2018, 260, 101-111.

8. D. Liu, P. Shi, W. Ren, Y. T. Liu, G. Niu, M. Liu, N. Zhang, B. Tian, W. X. Jing, Z. D. Jiang and Z. G. Ye, J. Mater. Chem. C., 2018, 6, 3206-3211.

9. K. Kalantari, A. M. Afifi, H. Jahangirian and T. J. Webster, Carbohydr. Polym., 2019, 207, 588-600.

10. H. Li, Y. Yang, F. F. Xu, T. X. Liang, H. R. Wen and W. Tian, Chem. Commun., 2019, 55, 271-285.

11. L. Matejka, M. Spirkova, J. Dybal, J. Kredatusova, J. Hodan, A. Zhigunov and M. Slouf, Chem. Eng. J., 2019, 357, 611-624.

12. L. Guadagno, L. Vertuccio, C. Naddeo, E. Calabrese, G. Barra, M. Raimondo, A. Sorrentino, W. H. Binder, P. Michael and S. Rana, Compos. Pt. B-Eng., 2019, 157, 1-13.

13. P. S. Tan, A. A. Somashekar, P. Casari and D. Bhattacharyya, Compos. Struct., 2019, 208, 367-376.

14. S. Nakagawa, S. Nakai, K. Matsuoka and N. Yoshie, Polymer, 2019, 161, 101-108.

15. A. Campanella, D. Dohler and W. H. Binder, Macromol. Rapid Commun., 2018, 39, 1700739

16. I. L. Hia, V. Vahedi and P. Pasbakhsh, Polym. Rev., 2016, 56, 225-261.

17. T. Szmechtyk, N. Sienkiewicz and K. Strzelec, Polym. Bull., 2018, 75, 149165.

18. S. R. White, N. R. Sottos, P. H. Geubelle, J. S. Moore, M. R. Kessler, S. R. Sriram, E. N. Brown and S. Viswanathan, Nature, 2001, 409, 794-797.

19. M. M. Diaz, J. Brancart, G. Van Assche and B. Van Mele, Polymer, 2018, 153, 453-463.
20. I. L. Hia, V. Vahedi and P. Pasbakhsh, Polym. Rev., 2016, 56, 225-261.

21. Y. L. Fang, X. S. Du, Z. L. Du, H. B. Wang and X. Cheng, J. Mater. Chem. A., 2017, 5, 8010-8017.

22. F. I. Altuna, J. Antonacci, G. F. Arenas, V. Pettarin, C. E. Hoppe and R. J. J. Williams, Mater. Res. Express., 2016, 3, 045003.

23. Y. T. Zou, L. Fang, T. Q. Chen, M. L. Sun, C. H. Lu and Z. Z. Xu, Polymers, $2018,10,474$

24. Z. Hu, Q. Shao, Y. D. Huang, L. Yu, D. Y. Zhang, X. R. Xu, J. Lin, H. Liu and Z. H. Guo, Nanotechnology, 2018, 29, 185602.

25. H. Ajam, P. Lastra-Gonzalez, B. Gomez-Meijide, G. Airey and A. Garcia, $J$. Test. Eval., 2017, 45, 1933-1940.

26. N. Roy, B. Bruchmann and J. M. Lehn, Chem. Soc. Rev., 2015, 44, 37863807.

27. P. Cordier, F. Tournilhac, C. Soulie-Ziakovic and L. Leibler, Nature, 2008, 451, 977-980.

28. C. Wang, N. Liu, R. Allen, J. B. H. Tok, Y. P. Wu, F. Zhang, Y. S. Chen and Z. N. Bao, Adv. Mater., 2013, 25, 5785-5790.

29. Y. G. Luan, X. A. Zhang, S. L. Jiang, J. H. Chen and Y. F. Lyu, Chin. J. Polym. Sci., 2018, 36, 584-591.

30. V. Kozik, A. Bak, D. Pentak, B. Hachula, K. Pytlakowska, M. Rojkiewicz, J. Jampilek, K. Sieron, J. Jazowiecka-Rakus and A. Sochanik, J. Nanosci. Nanotechnol., 2019, 19, 2489-2492.

31. X. D. Wang, X. H. Liu, H. Y. Yuan, H. Liu, C. T. Liu, T. X. Li, C. Yan, X. R. Yan, C. Y. Shen and Z. H. Guo, Mater. Des., 2018, 139, 372-379.

32. D. C. Lin, Y. Y. Liu, Z. Liang, H. W. Lee, J. Sun, H. T. Wang, K. Yan, J. Xie and Y. Cui, Nat. Nanotechnol., 2016, 11, 626-632.

33. C. F. Wang, M. Zhao, J. Li, J. L. Yu, S. F. Sun, S. S. Ge, X. K. Guo, F. Xie, B. Jiang, E. K. Wujcik, Y. D. Huang, N. Wang and Z. H. Guo, Polymer, 2017, 131, 263-271.

34. Y. X. Xu, W. J. Hong, H. Bai, C. Li and G. Q. Shi, Carbon, 2009, 47, 35383543.

35. W. S. Hummers Jr, R. E. Offeman, J. Am. Chem. Soc, 1958, 80, 1339.

Publisher's Note Engineered Science Publisher remains neutral with regard to jurisdictional claims in published maps and institutional affiliations. 